\title{
Pituitary metastasis from adenocarcinoma
}

\author{
Carolina Garcia Soares Leães, Luiza A.D. Silva, Júlia Fernanda Semmelmann Pereira-Lima, \\ Jorge Kramer, Miriam da Costa Oliveira' ${ }^{1}$
}

Metastatic tumors of the pituitary gland are very rare, representing only $1 \%$ of the pituitary mass submitted to surgeries, and are frequently related to primary neoplasia of breast and lung ${ }^{1,2}$.

\section{CASE}

A 50-year-old male patient sought medical care complaining of cephalalgia for 4 months, diplopia and ptosis of the left eyelid for 30 days. He reported loss of libido and erectile dysfunction in the previous six months. He was before a salutary individual. Physical examination: body mass index (BMI) 24, with ptosis and ophthalmoplegia of the left eye, arterial pressure of $140 / 80 \mathrm{mmHg}$. No other alterations.

Magnetic resonance imaging (Figure) showed heterogeneous mass into the sella turcica with suprasellar extension, causing displacement of optic chiasm and third ventricle floor, with intense opacification after contrast.

The laboratory exams showed normal thyroid function, low levels of gonadotropins and total testosterone, normal levels of basal cortisol and IGF-1 and very small increase in serum prolactin. Renal function, serum sodium and urine density were within normal limits. Central hypogonadism and slight hyperprolactinemia were detected, without evidence of posterior pituitary alteration.

An endoscopic transsphenoidal pituitary surgery were performed and anatomopathological exam showed metastasis of moderately differentiated adenocarcinoma of unknown primary site. Postoperative exams showed anemia, increased GGt, FA and CA 19-9. Immunohistochemical analysis indicated origin in the gastrointestinal tract, imaging of thorax and abdomen showed lymphadenopathies and small irregularities on the antral mucosa wall of the stomach. Upper gastrointestinal endoscopy with biopsy showed stomach antral adenocarcinoma.

\section{DISCUSSION}

Metastatic tumors of the pituitary gland are rare complications ${ }^{1}$ that occur between 1 and $3.6 \%$ of the patients with malign tumors ${ }^{2}$ and represent between 0.14 and $28.1 \%$ of the brain metastases ${ }^{3}$.

The metastasis reported here is from a gastric adenocarcinoma. In literature, the primary neoplasias more commonly associated with pituitary metastases are breast and lung cancer, corresponding to $30-47 \%$ and $19-$ $30 \%$ of all cases, respectively. Other sites were reported with lower frequency ${ }^{1-5}$.

The patient in question had no previous malignant history, in agreement with literature data which state that metastases may be the first manifestation of a malign tumor $^{1,2}$. Pituitary metastases are symptomatic in only $7 \%$ of the cases ${ }^{1,2}$, with predominance of Diabetes insipidus $(D I)^{3-5}$. This is an important characteristics in the differential diagnosis of invasive sellar lesions, as DI occurs in less than $1 \%$ of non-functioning pituitary adenomas in the first manifestation ${ }^{2}$. The patient in question did not present any clinical and laboratorial evidence of DI.

Pituitary metastases may have symptoms caused

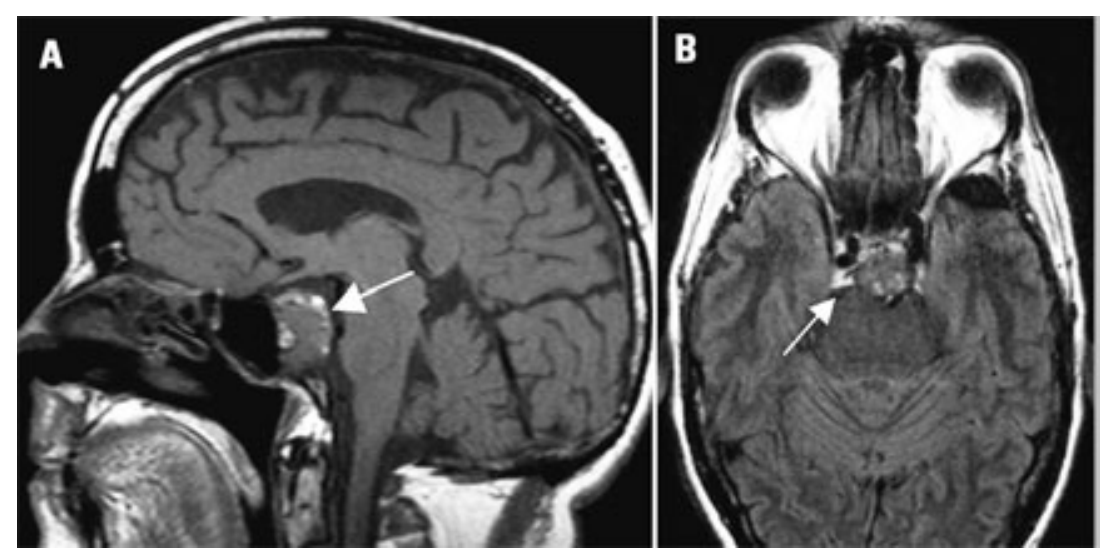

Figure. Magnetic resonance imaging of the head showing voluminous sellar lesion with suprasellar extension and optic chiasm displacement, lateral view in [A] and axial view in [B] (arrows). 
by the tumor mass, such as anterior pituitary dysfunctions, visual field alterations, cephalalgia and ophthalmoplegia $^{1-4}$, the last two present in this patient. The association of ophthalmoplegia and cephalalgia is described as a sign of probable metastasis ${ }^{5}$.

Pituitary metastases are rare, but should be included in the differential diagnosis of invasive sellar lesions, especially if associated with ophthalmoplegia, ptosis and DI.

\section{REFERENCES}

1. Komninos J, Vlassopoulou V, Protopapa D, et al. Tumors metastatic to the pituitary gland: case report and literature review. J Clin Endocrinol Metab 2004;89:574-580.

2. Fassett DR, Couldwell WT. Metastases to the pituitary gland. Neurosurg Focus 2004;16:E8.

3. Hoellig A, Niehusmann P, Flacke $S$, Kristof RA. Metastasis to pituitary ade- noma: case report and review of the literature. Cen Eur Neurosurg 2009; 70:149-153.

4. Bhatoe HS, Badwal S, Dutta V, Kannan N. Pituitary metastasis from medullary carcinoma of thyroid: case report and review of literature. J Neurooncol 2008;89:63-67.

5. Morita A, Meyer FB, Laws ER Jr. Symptomatic pituitary metastases. J Neurosurg 1998;89:69-73.

\section{METÁSTASE HIPOFISÁRIA DE ADENOCARCINOMA}

Neuroendocrinology Center of Santa Casa de Porto Alegre, Universidade Federal de Ciências da Saúde de Porto Alegre, Porto Alegre RS, Brazil.

Correspondence: Carolina Garcia Soares Leães - Rua Faria Santos, 142 - 90670 150 Porto Alegre RS - Brasil. E-mail: dracarolina@cisa.med.br

Conflitcs of interest: The authors report no conflict of interest

Received 23 May 2011, received in final form 28 June 2011. Accepted 6 July 2011

\title{
Seizure-like activity during etomidate-modified Wada test interfere with the evaluation of cognitive functions?
}

\author{
Pedro André Kowacs ${ }^{1}$, Tobias Alécio Mattei² , Carlos Rodrigo Goulart², \\ Samantha F. Blattes da Rocha ${ }^{3}$, Ricardo Ramina ${ }^{4}$, Cristiane A. Simão ${ }^{3}$, Murilo Sousa de Meneses ${ }^{5}$
}

The intracarotid amobarbital procedure (IAP), known as "Wada-test" is a useful tool in the investigation of patients who are candidates for temporal lobectomy or amygdala-hippocampectomy. Etomidate is a viable alternative to amobarbital. However, may induce epileptiform activity. We report on the emergence of irritative activity in epilepsy surgery candidates, during a modified Wada procedure using etomidate, and discuss this finding based on available literature ${ }^{1-4}$.

\section{CASE}

Case 1 - 35-year-old female with uncontrolled secondarily generalized seizures since the age of 14 years. Her magnetic resonance images (MRI) revealed right hippocampus sclerosis, and her video-EEG disclosed left-sided temporal interictal intermittent delta activity (TIRDA) and spiking, and an epileptogenic zone at the anterior aspects of the left temporal lobe. On the e-SAM shortly after etomidate injection on the right internal carotid artery there was slowing of the EEG tracing on the right hemisphere, associated with repetitive rhythmic sharp theta activity (Fig 1A). The patient remained hemiplegic on the contralateral side for four minutes, and the EEG recovered its original features 1.5 minutes after motor strength recovery (Fig 1B). When the study was performed on the left side the same abnormal features on EEG tracing were observed right after injection (Fig 1C). Memory and language cognitive tests were performed on both sides under abnormal EEG tracings and there were no signs of compromise of the neuropsychological evaluation as a consequence of the observed irritative EEG activity triggered by etomidate.

Case 2 - A 58 years-old female, suffered from seizures since the age of 34 years. Her MRI had disclosed a right-sided hippocampus sclerosis. An interictal SPECT had revealed a left temporal lobe hypoperfusion. Her Video-EEG recordings showed a right temporal interictal intermittent delta activity, and the recorded seizures revealed an anterior right temporal lobe epileptogenic zone. After etomidate injection on the left side, a slowing of the EEG background activity with associated TIRDA activity ensued on the ipsilateral hemisphere (Fig 2A). The patient remained hemiplegic on the contralateral side and aphasic for 7.5 minutes. Her EEG tracing, however, remained abnormal for a further minute after motor strength and speech normalization. When etomidate was injected in the right side an ipsilateral irritative activity was observed (Fig 2B) and remained during the 